\title{
Reinforced Laryngeal Mask Airway in ENT Surgery: a 15 years experience
}

Torres S., Sousa M., Pacheco M., Afonso D., Leão P., Gomes L.

Centro Hospitalar Entre Douro e Vouga, Departamento de Anestesiologia, Santa Maria da Feira, Portugal

BACKGROUND AND GOAL OF STUDY

The use of the reinforced laryngeal mask airway (rLMA) for ENT surgery has grown in the last decades. Several studies have reported a low incidence of respiratory complications and a smoother emergency with the use of this supraglottic device. However, there is still some concerns regarding the risk of an unprotected airway shared with the surgeon. Given the experience of our department, the authors propose to review the casuistry of the last 15 years.

\section{MATERIALS AND METHODS}

We retrospectively analyzed 19000 anesthetic records of elective ENT surgeries performed between January 2001 and December 2015. The procedures in which rLMA was used as the first choice for airway device were selected. We recorded the type and duration of surgery as well as the perioperative complications.

\section{RESULTS AND DISCUSSION}

rLMA was the airway device used in $47.8 \%$ of surgeries. The use of rLMA was more frequent for short-term surgeries as compared with long-term procedures, namely oropharynx and nose versus ear surgery (table 1). Adenotonsillectomy and septoplasty made up for $19,2 \%$ and $16,7 \%$ of the surgical procedures, respectively.

The incidence of perioperative complications was similar to that reported in literature ${ }^{(1,2)}$. Airway obstruction by the BoyleDavis gag was the most frequent complication $(4,5 \%)$, which, in most cases, was solved by switching to a larger blade (figure 1). Cough $(1,7 \%)$, laryngospasm $(0,5 \%)$ and airway soiling $(0,5 \%)$ were also observed. There was a low incidence of cases in which it was necessary to replace the rLMA for a tracheal tube $(0.9 \%)$. There were no reports of pulmonary aspiration.
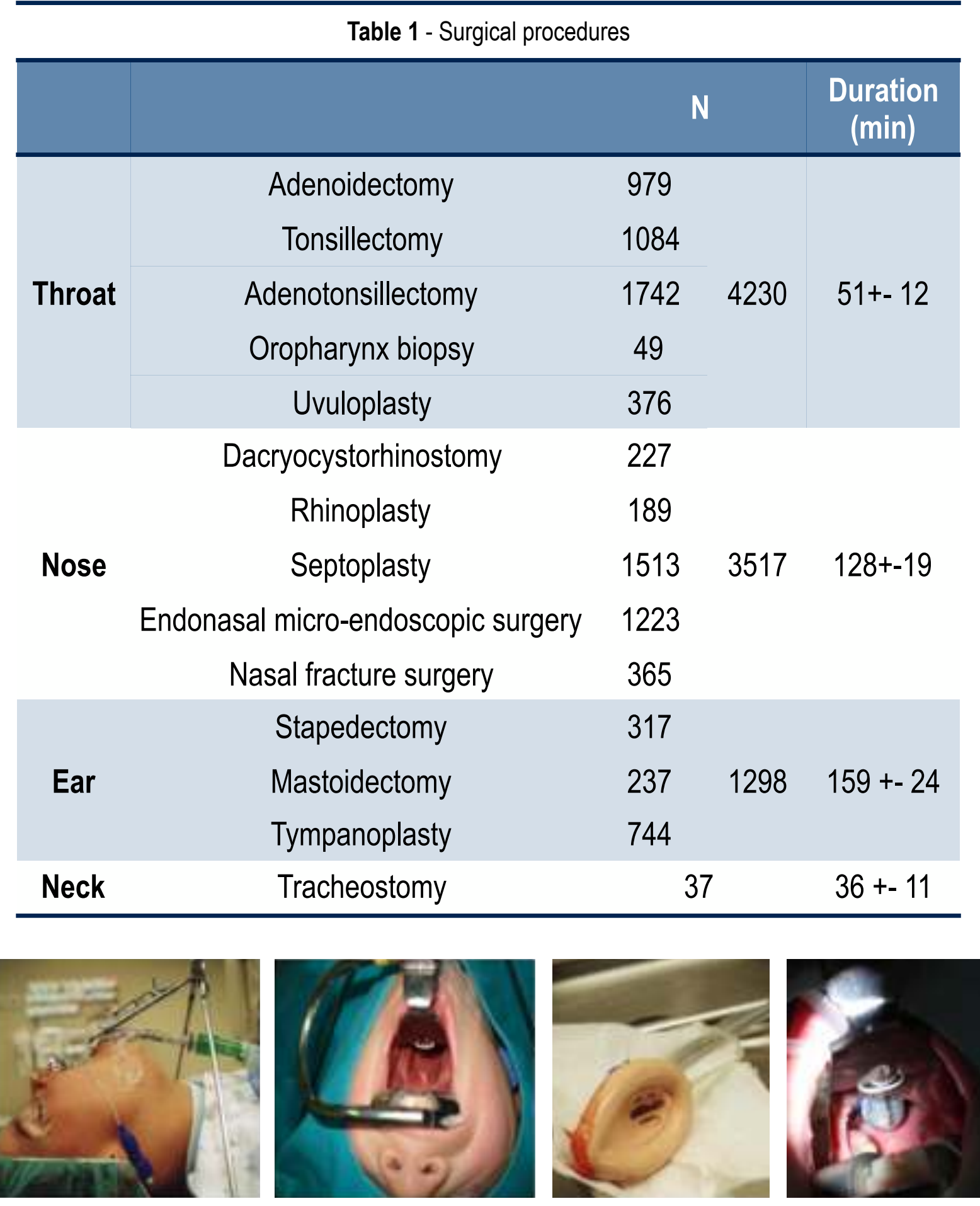

Figure 1 - rLMA use in ENT surgery

CONCLUSION

This retrospective study shows that rLMA could be a safe and effective alternative to orotracheal tube in ENT surgery. Its use requires a thorough periorperative approach of the airway, an accurate selection of patients and a close cooperation between the anesthetic and surgical team ${ }^{(3)}$.

\section{REFERENCES}

Eur J Anesthesiol 2008;25:261266

2. Surgery Curr Res 2015:5:223

3. Laryngoscope $2011 ; 121: 16201626$ 